Document downloaded from:

http://hdl.handle.net/10251/177800

This paper must be cited as:

Basterrechea-Chertudi, DA.; Rocher-Morant, J.; Parra-Boronat, L.; Lloret, J. (2020). Development of Inductive Sensor for Control Gate Opening of an Agricultural Irrigation System. IEEE. 250-255. https://doi.org/10.1109/FMEC49853.2020.9144810

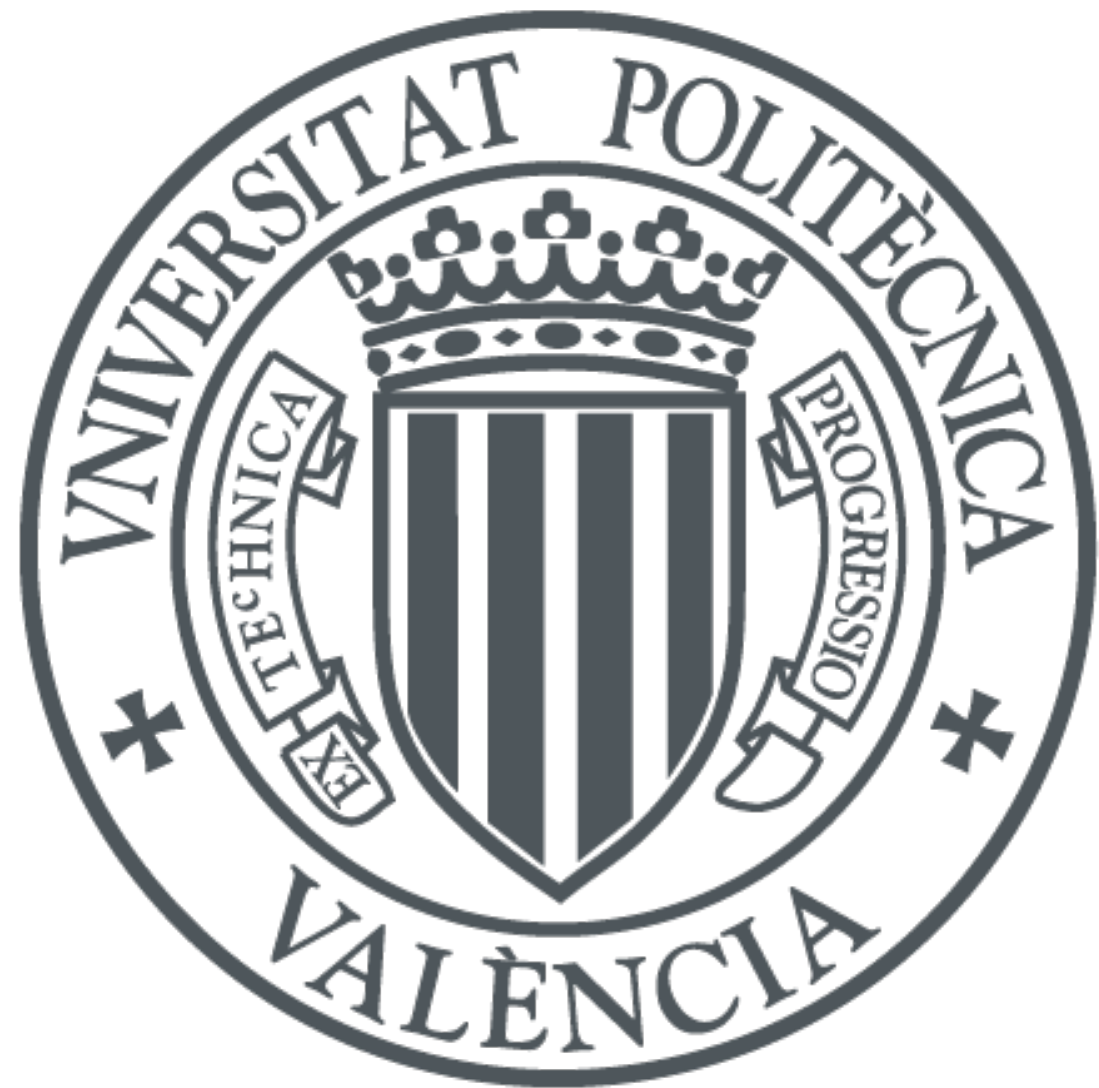

The final publication is available at

https://doi.org/10.1109/FMEC49853.2020.9144810

Copyright IEEE

Additional Information

(C) 2020 IEEE. Personal use of this material is permitted. Permission from IEEE must be obtained for all other uses, in any current or future media, including reprinting/republishing this material for advertising or promotional purposes, creating new collective works, for resale or redistribution to servers or lists, or reuse of any copyrighted component of this work in other works. 


\title{
Development of Inductive Sensor for Control Gate Opening of an Agricultural Irrigation System
}

\author{
Daniel A. Basterrechea, Javier Rocher, Lorena Parra, Jaime Lloret \\ Instituto de Investigación para la Gestión Integrada de Zonas Costeras, \\ Universitat Politècnica de València. \\ Gandía, Valencia (Spain) \\ dabasche@epsg.upv.es, jarocmo@doctor.upv.es, loparbo@doctor.upv.es, jlloret@dcom.upv.es.
}

\begin{abstract}
The water level in an agriculture irrigation channel is very important in order to control the gates of these channels to provide the necessary water to the crops. In this way, WSN (Wireless Sensor Networks) have high relevance to obtain this kind of data. In this paper, we propose a sensor to measure the depth changes in irrigation channels to control the gates opening. It is connected to an Adafruit Feather HUZZAH based on ESP8266, which allows us to build a mobile edge computing system. The developed sensor is based in two coils, where the first is powered by a sinus-wave and the second is induced by the first one. The coils are made of PVC that has high resistance for corrosion and low price. Besides, we use copper as a conductive metal. We test two different configurations of coils. P1 has 5 spires for the powered coil and 10 spires for the induced coil. On the other hand, P2 has 40 spires for the powered coil and 80 spires for the induced coil. The two prototypes were coiled in 1 layer. Then, both sensors are tested using a glass bottle where the water column increased with the target to obtain the information of the depth. In both prototypes, the difference of voltage between the maximum and minimum studied depths is more or less the same, 4.46V for $P 1$ and $4.44 \mathrm{~V}$ for $\mathrm{P} 2$. Nevertheless, during the stabilization test, the P1 showed better adaptation for the turbulences than the P2. The P1 shows an oscillation of $0.48 \mathrm{~V}$ where the $P 2$ has a maximum fluctuation of $3.2 \mathrm{~V}$.
\end{abstract}

Keywords-Precision agriculture; inductive coils; level coils.

\section{INTRODUCTION}

Agriculture is responsible for the production of the food that we consume. This activity has suffered various revolutions due to the demands of increasing food production to feed the population. These revolutions have been increased production, but they also increased the adverse effects in the environment. The main are: (I) Use of pesticides, (II) Use of excessive fertilizers, (III) Use of a large quantity of water, (IV) Wrong soil tillage. Nevertheless, agriculture has positive effects on the environment and positive ecological impact on the biodiversity of some animals [1].

Regarding the water, different studies indicate that agriculture use $70 \%$ of the water used in the world and over $40 \%$ in many OECD countries [2]. The freshwater is a valuable and limited resource due to its availability. The unsustainable use of groundwater and water bodies united to the decrease of water by global warming endangers food security in the future. Different methodologies have been developed to reduce water use in agriculture. Some of them are: drip irrigation, the use of sensors, reduce the amount of irrigation water, reuse of wastewater, etc. The use of technology, methodology of watering, and study of the variability in crops is englobed in the definition of precision farming.
Precision farming can be defined as the management of variability of crops and animal performance to improve benefits and reduce environmental impacts [2]. Even with the advantages of precision farming, many farmers do not apply it. For fostering precision agriculture, it is essential to transfer this knowledge to farmers and boost their participation. In addition, larger farms are more likely to use new technologies than little farms [4]. It is foreseeable that the increase in food production will be linked with precision farming. Precisión farming beneficies the agriculture in two different ways. First, with the reduction of environmental impacts, increaseasing the sustainability of the agriculture. Second, by lowering costs and increasing revenues. Thus, greater economic margins are produced. The increase of benefits will help avoiding abandonment of fertile lands as it is happening in many developed countries.

One of the leading technologies used in precision farming is Wireless Sensor Networks (WSN). However, The WSNs need improvements in power saving energy and data security [5]. In [6], the authors presented an algorithm for the power energy-saving and fault tolerance of the WSN for precision farming. The WSNs are composed of a physical sensor that takes the measures, and a microcontroller that controls the physical sensor and transmits the data to an access point or Internet. On the Internet, the data is transmitted to the final user. Another available technology for precision farming is remote sensing. Nonetheless, this has important gaps that are inhibiting their use. The use of satellite or airplanes have problems with the high cost and the frequency of the data obtained. To solve the problem in the frequency of data obtained, we can use drones. The drones are cheaper than airplanes or satellites. However, weather conditions and their limited batteries prevent real-time control. These gaps make WSN the best monitoring option. The use of an inductive sensor has been demonstrated as an alternative for monitoring the conductivity of water. These can be used for detecting irregular values of conductivity that can be related to the illicit discharge [7]. However, as far as we know, the use of the inductive sensor for the monitoring water level in open channels has not been studied.

In this paper, we present a sensor for measuring the water level in the irrigation channel. We use the sensor with valves that will close or open the irrigation canal gates. The actuators will close the gate (in primary channel) when it is necessary to send water to a secondary channel (highest) and will open it when it is no longer required to carry more water. In addition, the level sensor is used to verify that the water effectively reaches the secondary channel. This sensor is based on two coils. One of the coils generates a magnetic field and the other one is induced. The coils are wired on PVC pipes which provides greater robustness to the sensor. 
In this way, when it is located in the channel, the sensor is not affected by the hitting of sediments or by other animals that can hit it.

The rest of the paper is structured as follows. The related works are presented in Section II. In Section III, we explain the test bench of the experiment. In Section IV, we show the structure for our system and the microcontroller used. The results and discussions are described in section V. Finally, in section VI, we explain the main conclusion and future work.

\section{RELATED WORK.}

In this section, several papers similar to our proposal are analyzed. First, they are discussed and later compared with our solution.

The inductive sensors have been presented previously to monitor the water level. Rocher et al. [8] presented a similar sensor for monitoring water levels inside pipes. In this paper, the authors defend that this sensor can be used for monitoring illegal dumping, obstruction, and leaks in pipes networks. The sensor consists of two coils (powered coil and induced coil) coiled over a semi-cylinder of PVC with 20 $\mathrm{mm}$ of diameter. They tested with different prototypes coiled in various forms and with a different number of spires. They concluded that prototyped with 55 spires in induced and powered coils was the best prototype. In this paper, the authors centered on pipes and they did not study its use in open channels. The prototypes tested are not suitable for monitoring open channels due to their semicircular shape. What causes them to be easily misadjusted because they have two separate pieces.

Another alternative is the use of video. Tauro et al. [9] proposed the use of large scale particle image velocimetry. This is a methodology for monitoring the surface flow in the environment with extreme floods. They used a GoPro Hero 3 and two green lasers located in a telescopic bar. The GoPro captures the image and with an algorithm detect floating elements, and estimate the speed of the water in an area according to the position of the floating elements frame to frame. The two lasers are used for calibrating the system with two fixed points. The video analyzers have two critical gaps that make them unsuitable. The first is the computation needs for processing the video. The second is the need for the water to transport an object. In many cases, the water does not transport any object that can be used for the camera to control the velocity.

Another alternative is presented by Michailovsky et al. [9]. They studied the use of Satellite radar altimetry (obtained to Earth and Planetary Remote Sensing Lab (EAPRS)) for monitoring the surface water in the Zambezi River basin. The objective was to calculate the discharge of the rivers in the basin. They studied three scenarios (I) with an in situ rating curve available, (II) With one simultaneous field measurement of cross-section and discharge, (III) with historical discharge data available, These scenarios have been compared with the values of the real discharge. They obtained that the first method has an error between 4.1 to 6.5 $\%$, while the other methods have an error of 6.9 to $13.8 \%$. The principal gap in the use of satellite images is the frequency of the obtained data. The satellites have a return time to the same point, which can go from a few weeks to months. This makes them a lousy method for continuous data collection. In addition, the article mentioned that due to the precision of the satellite image, it could not be used in rivers with a width of fewer than $80 \mathrm{~m}$.

The electrical conductivity of the water can be used for evaluating the water level in an area or tank. HernándezNolasco et al. [11] developed a system for floods based on the electrical conductivity of the water. The system consists of a microcontroller Netduino Plus 2 to process and send the information. On the other hand, the sensing part consists of different open circuits that close when the water touches the two ends of the loop. The circuits consist of a copper wire which is in the base and secondary copper wires, which are located at different heights. When any of the circuits closes, it sends the information to an access point, and from this to a computer. If it is necessary the computer processes the information and sends an alarm to the smartphones of the population. This method can be used in the case we want to set a threshold value. The proposed system only tells us when the water has reached a point. Nevertheless, this system does not give us information on the amount of water between points.

Capacitive sensors are another alternative for measuring water levels. Reverter et al. [12] used a capacitive sensor for monitoring the water level in a metallic tank. The sensor has two electrodes where one is insulated. The water level can be calculated due to the change on the difference between the dielectric value of air and water. This type of sensors is not a solution for open channels. Due to this, we need to place sensors with a height equal to the height we want to control. In addition, the presence of sediments or rocks, which are deposited in the lower parts of the electrode can affect the measurement.

Other types of sensors are based on acoustic or light reflection. These sensors emit light or acoustic waves that are reflected in the surface of the water. However, these methods present problems when making measurements in areas with high agitation or with the presence of waves. For solving this problem, $\mathrm{Li}$ et al. [13] proposed the use of multiple-input multiple-output ultrasonic transducers. They used four transmitting elements and four receiving elements. They achieved an improvement in measurement accuracy. The main problem with these types of sensors is that in nature, the differences distance between the emitter and the receiver can be modified due to the presence of animals. This causes the sensor cannot be accurate.

Finally, the last alternative analyzed for measuring the water level is presented by Antonio-Lopez et al [14]. They showed an optical fiber sensor for measuring the water level. A multimode fiber without cladding was used as an optical fiber. The sensor works due to when the fiber is submerged in a liquid the refractive index is different depending on how submerged it is. They concluded that the more the fiber is submerged in water, the more the peak wavelength will be shifted to is longer wavelengths. This type of sensor has the same gaps that we explained for capacitive sensors.

In this paper, we present an inductive sensor for monitoring the water column in irrigation open channels. We develop the sensor with two coils (powered and induced coil). The sensor can be used for continuous monitoring. One of the characteristics of our sensor is that it can be used for continuous monitoring. Stability is another characteristic of our sensor. It can be observed in the results section. 
Furthermore, this type of sensors is very robust thanks to its PVC structure.

\section{TEST BENCH}

In this section, the prototypes that have been used and the necessary devices to carry the experiments are described. The test bench is segmented in two different subdivisions.

\section{A. Prototype characterization}

We developed different prototypes that measure the depth of the water in rivers to improve the river modeling techniques using low-cost sensors. The prototypes were created using different sizes of PVC (polyvinyl chloride) material, consuming $2.2 \mathrm{~cm}$ long and $1.7 \mathrm{~cm}$ of diameter for the P1. Besides, $5.2 \mathrm{~cm}$ long and $2.5 \mathrm{~cm}$ of diameter were needed for the P2. Copper wire was used to coil the different prototypes. The P1 was coiled using $0.2 \mathrm{~mm}$ thickness copper. Nevertheless, a $0.4 \mathrm{~mm}$ copper coil was employed for the $\mathrm{P} 2$.

Otherwise, the P1 and P2 were distinguished in two different sides, the Powered Coil (PC) and Inductive Coil (IC). The P1 was designed with ten winds for the PC part and five winds for the IC, likewise, 40 winds for PC and 80 winds for IC were used in $\mathrm{P} 2$, this information is represented in Table 1.

TABLE 1. DEVELOPED PROTOTYPES.

\begin{tabular}{|c|c|c|}
\hline Prototype & P1 & P2 \\
\hline Image & & \\
\hline Spires of PC & 10 & 40 \\
\hline Spires of IC & 5 & 80 \\
\hline Layers & 1 & 1 \\
\hline
\end{tabular}

The two tested coils are represented in Figure 1 . The prototypes were coiled against the hands of the clock, trying to make the spires as close as possible, to obtain the best results. Besides, the $\mathrm{P} 1$ and $\mathrm{P} 2$ were powered in the same direction- In this case, upside-down of the coiled way, using the clockwise direction. This helps to maintain a similar basis for all prototypes and to obtain more relevant data.

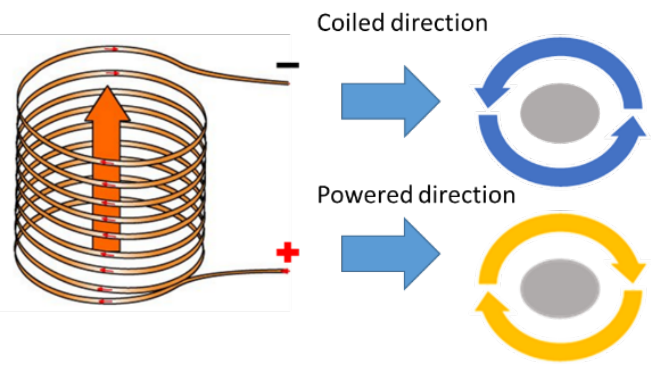

Figure 1. Coiled direction and powered direction.

\section{B. Performance of the experiment}

We powered the coils in a clockwise direction, using the end of the coil as a negative part or ground reference. Besides, we applied 10Vpp (voltage peak to peak) to feed the P1 and P2 using the generator AFG 1022 [15]. Then we measured the output voltage (Vout) using the oscilloscope TBS1104. [16]

The circuit we have used is composed of a resistance of 47 Ohms serially located to the coil, to reduce electromagnetic noise and obtain a reliable value of Vout. Moreover, the induced part of the circuit has a capacitator of $10 \mathrm{nF}$ in parallel to capture the energy and maintain the magnetic field.

The experiment was done using a glass bottle where measure marks were drawn every $2.5 \mathrm{~cm}$, from 0 to $15 \mathrm{~cm}$. The glass has a height of $16.2 \mathrm{~cm}$ and $8 \mathrm{~cm}$ of diameter. Following, the coil was placed suspended between two different marks. The P1 was located among $5 \mathrm{~cm}$ and $7,5 \mathrm{~cm}$ and P2 between $5 \mathrm{~cm}$ and $10 \mathrm{~cm}$. Once the coil was placed, we began to fill the bottle with water measuring the Vout for each mark. The water volume that we use is $1 \mathrm{~L}$.

Finally, we test the stability of the P1 and P2. For this, we put each coil into the glass bottle, and we fill the bottle up to the mark of $15 \mathrm{~cm}$. Then, we introduce the sensor between $5 \mathrm{~cm}$ and $7.5 \mathrm{~cm}$, in the case of $\mathrm{P} 1$ and $5 \mathrm{~cm}$ and $10 \mathrm{~cm}$ for P2. Following, a tool was used to shake the sensor with intensity, changing the variation of movements to simulate the real environment of a irrigation channel.

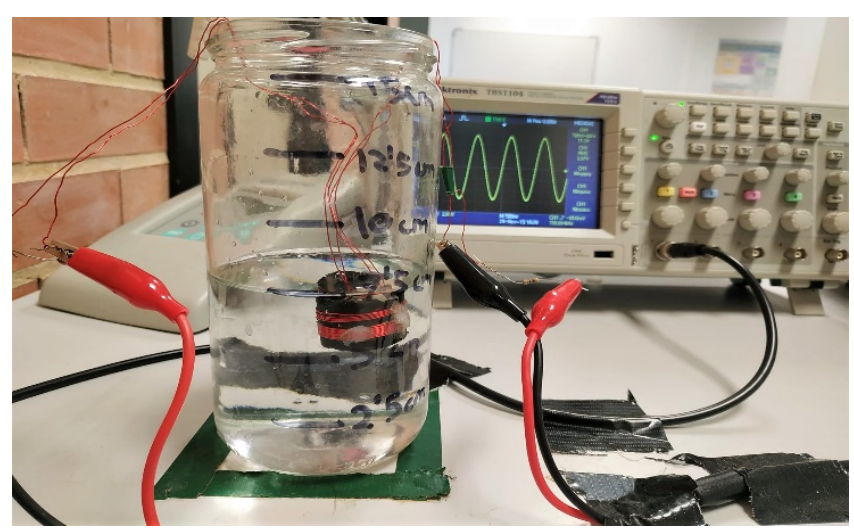

Figure 2. Experimental setup.

\section{SYSTEM PROPOSAL}

In this section, the proposed WSN is presented. For reaching this target, our system is going to base in inductive sensors for measuring the changes in depth of water columns in irrigation channels. The objective of these sensors is to indicate when it is necessary to open or close the gates of this channel. This is very important to control a sustainable irrigation in agriculture.

Additionally, this type of sensor is to be part of the European Project SMARTWATIR (Smart wireless sensor network to detect and purificate water salinity and pollution for agriculture irrigation). The sensor is going to be part of more prototypes that will be measuring different parameters for detecting and monitoring pollutants in agriculture irrigation.

A. WiFi node

In this subsection, the used wireless node is presented. The function of the node is to obtain and gather data from the 
sensors. Then, the induced voltage is transformed into water level values, and it is sent the data wirelessly.

Four the WSN, the used node is the Adafruit Feather HUZZAH based on ESP8266, see Figure 3. This is one of the best nodes for this system because of the low cost, easy to program (it can be programmed using IDE Arduino), one analog input of $1.0 \mathrm{~V}$ máx, and $3.3 \mathrm{~V}$ regulator with $500 \mathrm{Ma}$ peak current output [17].

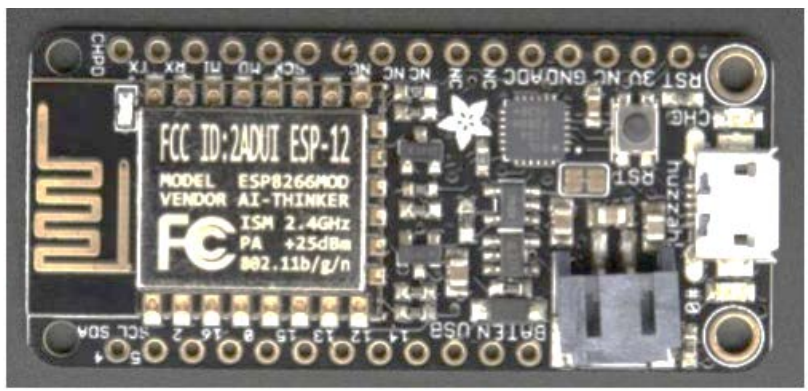

Figure 3. Adafruit Feather HUZZAH ESP8266 development board.

The propotypes were connected to the analog input of the Adafruit Feather HUZZAH (fith pin starting from the right at the bottom of the board shown in Fig. 3).

\section{B. Deployment and architecture.}

In this subsection, we present the deployment of the developed system, including the nodes, the function, and the used architecture.

Our proposal is based on controlling the changes in the water column in an agriculture irrigation system to open or close the gates and provide the necessary water to the crops. These sensors are going to be with other sensors that detect pollutants in agricultural irrigation.

Figure 4 represents the deployment of the sensors in the irrigation channel for agriculture. The channel has a grid, which is required to remove bulky material to the water flow. Behind the grid, four depth sensors are placed each one in a different profundity to obtain the best accuracy as possible. Then, the turbidity sensor is situated. This last sensor is part of the sensors for pollution tracking, but this paper is focused on the depth sensors. Likewise, the used sensors are collocated inside of a watertight box. This box is partially buried in the soil near the irrigation channels. Inside of this case will place the node, the battery, and the communication module. Data will be sent through WiFi. A pipe connects this container with the channel where the sensor is collocated in the water. Moreover, this system will use a solar panel to charge the battery.

In this case, it will not be necessary to replace the power, and we eliminate the requirement of maintenance. Therefore this sensor is going to be more sustainable exploiting solar energy.

Figure 5 displays the architecture of the WSN. The sensors get the electrical values of the difference in water level. The nodes transformed the electrical values in to depth values. Likewise, the nodes are provided with WiFi that is used to send data wirelessly. Additionally, these data are stored. Then, artificial intelligence is applied for decision making decisions when it is necessary to open or close the gates in the irrigation channel.

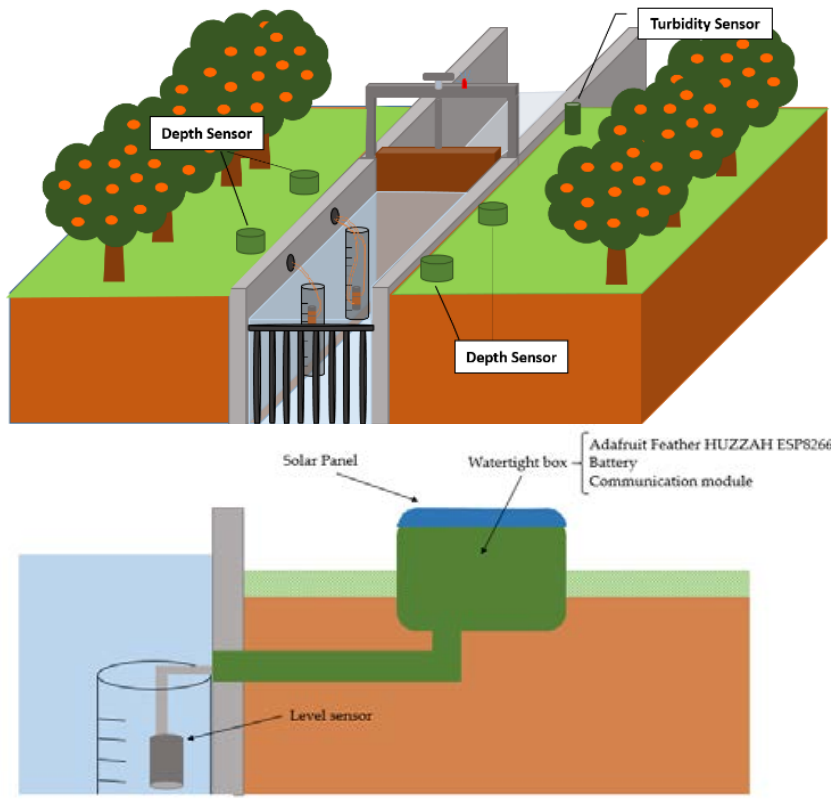

Figure 4. Deployment of the sensors in the Irrigation channels for agriculture.

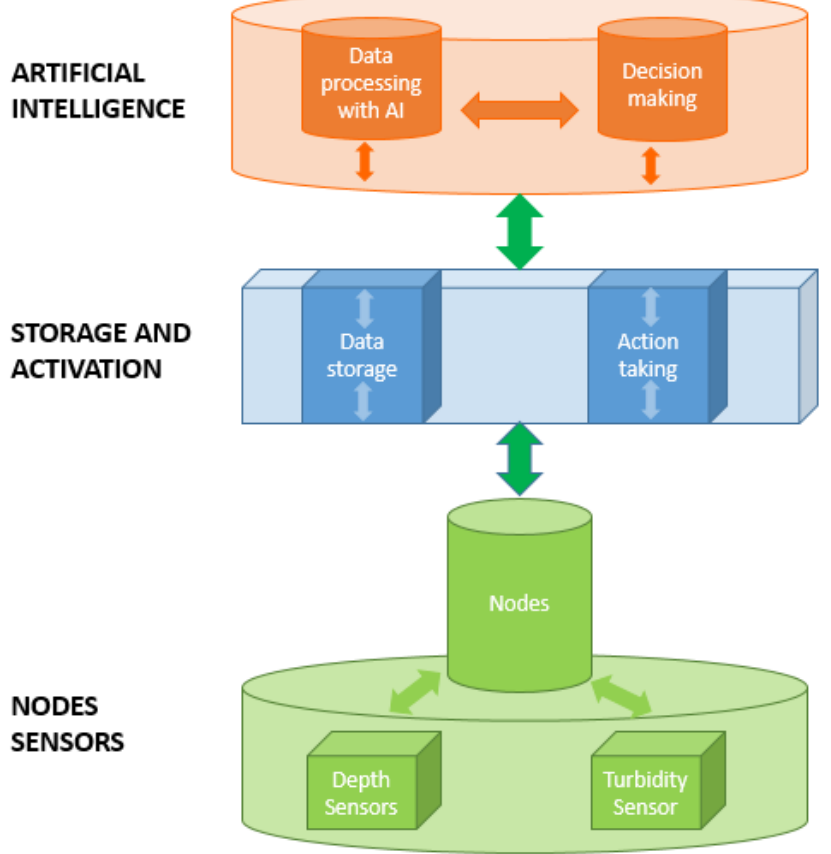

Figure 5. Architecture of the WSN.

\section{RESULTS}

In this section, we detail the results of the prototypes that we have used for the measurements. First, the test of each prototype with a different quantity of water are detailed. Successive, the prototypes are used to verify the stability of the measures that are obtained by the sensors. Finally, the best sensor is tested for in different water column position.

\section{A. Depth Tests}

First of all, the working frequency (WF) was found. The WF for P1 was detected at $770 \mathrm{kHz}$ and for P2 in $130 \mathrm{kHz}$. Following, the P1 was located between the marks $5 \mathrm{~cm}$ and $7.5 \mathrm{~cm}$, and the glass bottle starts to fill each $2.5 \mathrm{~cm}$. Then, the same was done for the P2. 
The P1 shows an initial Vout of $14.18 \mathrm{~V}$ for $0 \mathrm{~cm}$ depth and a final Vout of $9.72 \mathrm{~V}$ that exposed the tendency of voltage to decrease as the water layer increases. However, the $\mathrm{P} 2$ represents the opposite situation, where the initial Vout is $23.80 \mathrm{~V}$ and the final is $28.23 \mathrm{~V}$. In this situation, the voltage growth with the increase of the water layer. The two prototypes behave differently each other because the magnetic field that is produced by each one is not the same. Additionally, the performance of the magnetic coil is closely related to the structure of the prototypes.

In Figure 6 is displayed the model adjusts for each prototypes using a eureqa and statgraphics software. [18], [19]. The Vout of the sensors is contrasted with the depth of the different marks of the glass bottle. Likewise, the mathematical models of the two kinds of prototypes are shown in Eq. (1) and Eq. (2). Wehre $V$ is the Vout in volts and $D$ is the Depth in $\mathrm{cm}$.

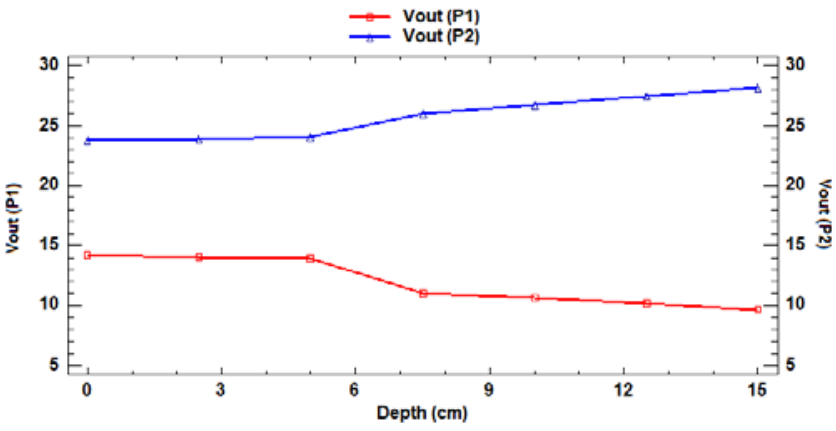

Figure 6. Model of depth changes

$$
\begin{gathered}
\mathrm{V}=14.248-8.030 \times e^{-8.592 / D)} \\
\mathrm{V}=23.82+0.03 \times \mathrm{D}+0.26 \times D \times e^{-14582 / 0.2^{\wedge} D}
\end{gathered}
$$

The correlation obtained between the values is very high being the R2 for the P1 of 0.923 and 0.999 for the P2. This is a statistical parameter that indicates the adaptation of the model for each measured point.

\section{B. Stability Tests}

After obtaining the mathematical model, the stability of the sensors was tested. This is necessary to choose the best prototype that will beused in the field. However, the signal of the sensor must remain stable in a hard condition. To ensure that the current of the irrigation channel does nott affect the data, contributing like this to have reliable values.

To test the stability of the signal, we shake very hard the prototypes. Likewise, the standard desviation between the real value and measured value in the experiment of $\mathrm{P} 1$ are represented in Figure 7. Besides, in Figure 8 are discussed the results of the P2.

The sensors were shacked for 1 minute, and we took the signal value every 1 second. The P1 demonstrates very high adaptability for the hard conditions of the test. Furthermore, we have started with a $9.2 \mathrm{~V}$ that is represented as a point 0 . Once we have finished the test, we obtained the maximum change of $0.24 \mathrm{~V}$. This shows that the P1 has a good stability. In the case of P2, the original value was $28.2 \mathrm{~V}$, this is represented as point 0 . In the highest changes the values turn to $2.6 \mathrm{~V}$. Figure 8 displayed very well how the values of P2 are very far of the initial values in all of the experiment.

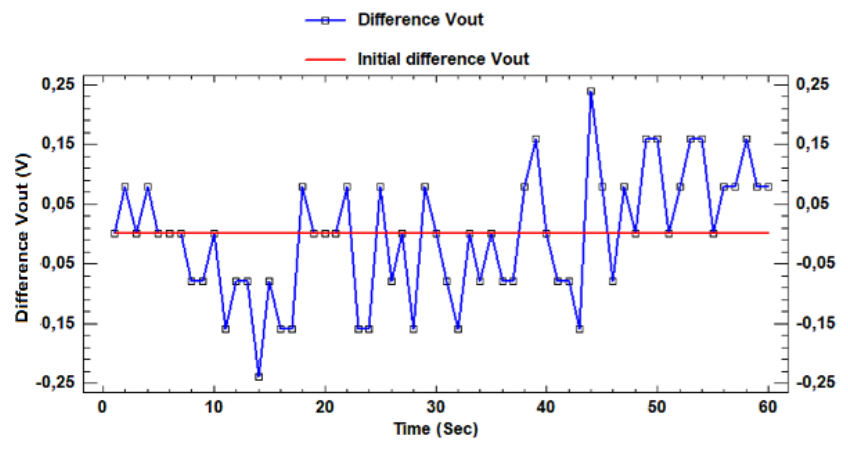

Figure 7. Stability of the P1.

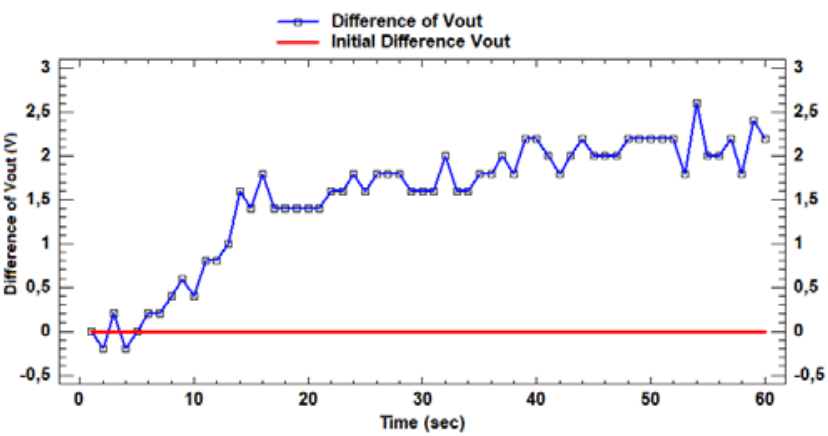

Figure 8.Stability test of the P1.

Likewise, the frequency of the values, or histogram, from the stability tests are displayed in Figures 9 and 10. In P1 we can appreciated that the highest values are around the initial value in the range of $9.1 \mathrm{~V}$ and $9.3 \mathrm{~V}$. Besides, he maximum frequency of values is found in this range of data. Besides, the P2 has the maximum part of the values far away from the starting value, where most of the data are found between $26 \mathrm{~V}$ and $27 \mathrm{~V}$. This means that the highest frequency of the data is placed below the started value, displaying changes when the turbulence is high. Moreover, the obtained values were analysed in Table 2.

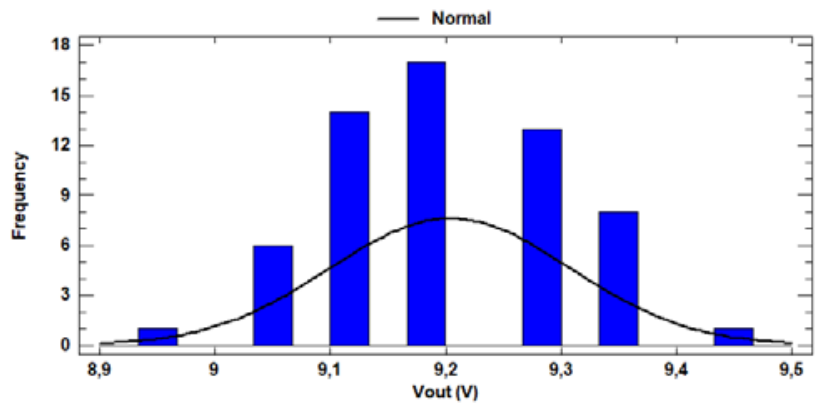

Figure 9. Distribution frequency of values in P1.

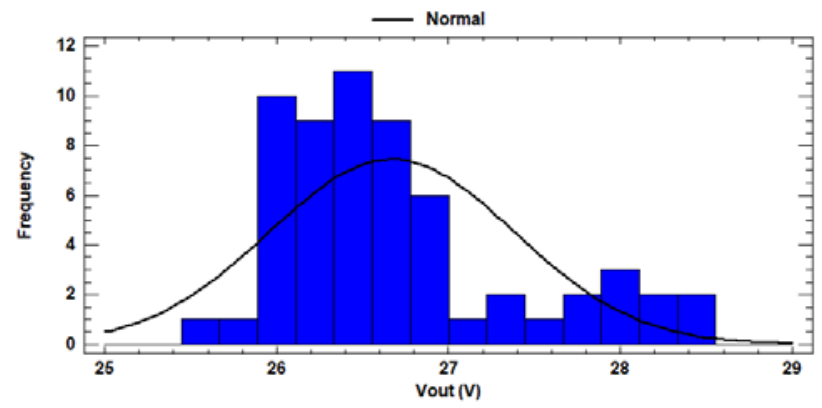

Figure 10. Distribution frequency of values in P2. 
TABLE 2. ANALYSE OF STABILITY VALUES.

\begin{tabular}{|l|c|c|}
\hline & P1 & P2 \\
\hline Count & 60 & 60 \\
\hline Standard Desviation & 0,104 & 0,713 \\
\hline Coefficient Variation & $1,14 \%$ & $2,68 \%$ \\
\hline Standard Error & 0,013 & 0,092 \\
\hline Standard Geometric Desviation & 1,011 & 1,026 \\
\hline Absolute Average Desviation & 0,008 & 0,020 \\
\hline Minimum & 8,96 & 25,60 \\
\hline Maximum & 9,44 & 28,40 \\
\hline Range & 0,48 & 2,80 \\
\hline
\end{tabular}

The highest difference of Vout in $\mathrm{P} 1$ is more or less $0,48 \mathrm{~V}$ and in the P2 is between $0,2 \mathrm{~V}$ and $3,2 \mathrm{~V}$. Besides, Table 2 displays that the $\mathrm{P} 1$ has a lower standard deviation than the P2. The error is also smaller in P1 with 0.013 compared to the error of $\mathrm{P} 2$, which is 0.092 . This means that the $\mathrm{P} 1$ shows the best operation in environmental conditions.

\section{CONCLUSION AND FUTURE WORKS}

In this paper, we have proposed the use of the inductive sensor to measure the depth in irrigation channels for agriculture. The proposed sensor aims to control when it is necessary to open or close the channel gates to irrigate the crops. It is connected to an Adafruit Feather HUZZAH building a WSN, and allowing us to create a mobile edge computing system. It brings many advantages to agriculture systems like the automation of the processes and sustainable use of resources. In this case, the control of the gates is necessary to adjust the use of water that is a very scarce resource nowadays.

Once we have performed the measurements, we determine that the P1 works with the lower values of voltage than the P2. Likewise, the P1 shows a maximum Vout of $14.18 \mathrm{~V}$ and a minimum of $9.72 \mathrm{~V}$. Besides, P2 shows the highest value of $28.4 \mathrm{~V}$ and the lowest date of $23.8 \mathrm{~V}$. Likewise, the P1 has a $4.46 \mathrm{~V}$ of difference between the $0 \mathrm{~cm}$ depth and $15 \mathrm{~cm}$ depth, where the P2 has a $4.44 \mathrm{~V}$. Otherwise, P1 has a better correlation $(\mathrm{R} 2=0.93)$ between them than the dates of P2 (R2=0.99.).

The stability of the prototypes was very different. In P1, the maximum difference between the values was $0.48 \mathrm{~V}$, which is very low, reversely of P2 that the change was the highest one with $3.2 \mathrm{~V}$. This proves that the prototype that works better in adverse conditions is the P1.

In future works, we will test more prototypes using another type of sensor to measure a more significant range of water column with better accuracy.

\section{ACKNOWLEDGMENT}

This work has been partially supported by European Union through the ERANETMED (Euromediterranean Cooperation through ERANET joint activities and beyond) project ERANETMED3-227 SMARTWATIR by the Conselleria de Educación, Cultura y Deporte with the Subvenciones para la contratación de personal investigador en fase postdoctoral, grant number APOSTD/2019/04, and through the "Ayudas para contratacion predoctoral de
Formación del Profesorado Universitario FPU (Convocatoria 2016)”. Grant number FPU16/05540.

\section{REFERENCES}

[1] M. Önder, E. Ceyhan, and A. Kahraman, "Effects of Agricultural Practices on Environment," in International Conference on Biology, Environment and Chemistry, 2011.

[2] OECD (2019, December), Water and agriculture. , [Online]. Available: https://www.oecd.org/agriculture/topics/water-and-agriculture/

[3] European commission, "Precision farming - EIP-AGRI - European Commission,” EIP-AGRI, Nov-2020. [Online]. Available: https://ec.europa.eu/eip/agriculture/en/digitising-agriculture/developingdigital-technologies/precision-farming-0. [Accessed: 11-Jan-2020].

[4] K. P. Paudel, M. Pandit, Mishra, Ashok K, and E. Segarra, "Why Don’t Farmers Adopt Precision Farming Technologies in Cotton Production?,” in Agricultural \& Applied Economics Association's 2011 AAEA \& NAREA Joint Annual Meeting, 2011.

[5] S. Ivanov, K. Bhargava, and W. Donnelly, "Precision Farming: Sensor Analytics,” IEEE Intelligent Systems, vol. 30, no. 4, pp. 76-80, Jul. 2015.

[5] L. Parra et al., "Design of a WSN for smart irrigation in citrus plots with fault-tolerance and energy-saving algorithms," Network Protocols and Algorithms, vol. 10, no. 2, p. 95, Jun. 2018.

[7] J. Rocher, D. A. Basterrechea, L. Parra, and J. Lloret, “A New Conductivity Sensor for Monitoring the Fertigation in Smart Irrigation Systems," $10^{\text {th }}$ International Symposium on Ambient Intelligence (ISAmI), 2019.

[8] J. Rocher, L. Parra, J. Lloret, and J. Mengual, “An Inductive Sensor for Water Level Monitoring in Tubes for Water Grids,” in 2018 IEEE/ACS 15th International Conference on Computer Systems and Applications (AICCSA), 2018

[9] F. Tauro, M. Porfiri, and S. Grimaldi, “Orienting the camera and firing lasers to enhance large scale particle image velocimetry for streamflow monitoring," Water Resources Research, vol. 50, no. 9, pp. 7470-7483, Sep. 2014.

[10] C. I. Michailovsky, S. McEnnis, P. A. M. Berry, R. Smith, and P. Bauer-Gottwein, "River monitoring from satellite radar altimetry in the Zambezi River basin,” Hydrology and Earth System Sciences, vol. 16, no. 7, pp. 2181-2192, Jul. 2012.

[11] J. A. Hernandez-Nolasco, M. A. W. Ovando, F. D. Acosta, and P. Pancardo, "Water Level Meter for Alerting Population about Floods," in 2016 IEEE 30th International Conference on Advanced Information Networking and Applications (AINA), 2016.

[12] F. Reverter, X. Li, and G. C. M. Meijer, "Liquid-level measurement system based on a remote grounded capacitive sensor," Sensors and Actuators A: Physical, vol. 138, no. 1, pp. 1-8, Jul. 2007.

[13] P. Li, Y. Cai, X. Shen, S. Nabuzaale, J. Yin, and J. Li, “An Accurate Detection for Dynamic Liquid Level Based on MIMO Ultrasonic Transducer Array," IEEE Transactions on Instrumentation and Measurement, vol. 64, no. 3, pp. 582-595, Mar. 2015.

[14] J. E. Antonio-Lopez, J. J. Sanchez-Mondragon, P. LiKamWa, and D. A. May-Arrioja, "Fiber-optic sensor for liquid level measurement," Optics Letters, vol. 36, no. 17, p. 3425, Aug. 2011.

[15] Tektronix. (2019, December), [Online]. Available: https://www.mouser.es/ProductDetail/Tektronix/AFG1022?qs=6oMev5NR ZMFfiXipKz6z9Q\%3D\%3D

[16] Tektronix. (2019, December), [Online]. Available: $\underline{\mathrm{h}}$ https://www.tek.com/oscilloscope/tbs1104

[17] Adafruit (2020, January), [Online]. Avaiable: https://www.adafruit.com/product/2821

[18] Eureqa (2019, December), [Online]. Available: https://www.nutonian.com/products/eureqa/

[19] Statgraphics Centurion XVIII (2019, December), [Online]. Available: https://statgraphics.net/ 